\title{
Testicular Pain
}

National Cancer Institute

\section{Source}

National Cancer Institute. Testicular Pain. NCI Thesaurus. Code C78636.

A painful sensation in the testis. 